\title{
Detection and Type-Distribution of Human Papillomavirus in Vulva and Vaginal Abnormal Cytology Lesions and Cancer Tissues from Thai Women
}

\author{
Jarunya Ngamkham*, Krittika Boonmark, Thainsang Phansri
}

\begin{abstract}
Vulva and Vaginal cancers are rare among all gynecological cancers worldwide, including Thailand, and typically affect women in later life. Persistent high risk human papillomavirus (HR-HPV) infection is one of several important causes of cancer development. In this study, we focused on HPV investigation and specific type distribution from Thai women with abnormality lesions and cancers of the vulva and Vaginal. A total of ninety paraffin-embedded samples of vulva and Vaginal abnormalities and cancer cells with histologically confirmed were collected from Thai women, who were diagnosed in 2003-2012 at the National Cancer Institute, Thailand. HPV DNA was detected and genotyped using polymerase chain reaction and enzyme immunoassay with GP5+/ bio $6+$ consensus specific primers and digoxigenin-labeled specific oligoprobes, respectively. The human $\beta$-globin gene was used as an internal control. Overall results represented that HPV frequency was 16/34 $(47.1 \%)$ and $8 / 20(40.0 \%)$ samples of vulva with cancer and abnormal cytology lesions, respectively, while, $3 / 5(60 \%)$ and $16 / 33(51.61 \%)$ samples of Vaginal cancer and abnormal cytology lesions, respectively, were HPV DNA positive. Single HPV type and multiple HPV type infection could be observed in both type of cancers and abnormal lesion samples in the different histological categorizes. HPV16 was the most frequent type in all cancers and abnormal cytology lesions, whereas HPV 18 was less frequent and could be detected as co-infection with other high risk HPV types. In addition, low risk types such as HPV 6,11 and 70 could be detected in Vulva cancer and abnormal cytology lesion samples, whereas, all Vaginal cancer samples exhibited only high risk HPV types; HPV 16 and 31. In conclusion, from our results in this study we suggest that women with persistent high risk HPV type infection are at risk of developing vulva and Vaginal cancers and HPV 16 was observed at the highest frequent both of these, similar to the cervical cancer cases. Although the number of samples in this study was limited and might not represent the overall incidence and prevalence in Thai women, but the baseline data are of interest and suggest further study for primary cancer screening and/or developing the efficiency of prophylactic HPV vaccines in Thailand.
\end{abstract}

Keywords: Human papillomavirus - vulva - vaginal - type distribution - Thai women

Asian Pac J Cancer Prev, 17 (3), 1129-1134

\section{Introduction}

Human papillomavirus (HPV) is a small nonenveloped, double-stranded DNA virus (de Villiers et al., 2004; Moody et al., 2010) that typically infects mucosal and cutaneous epithelial tissues (Ganguly, 2012; Bharti et al., 2010). More than 100 types of HPVs have been characterized, in which 30-40 types of these have been isolated from human lower-genital tract (de Villiers, 2001; zur Hausen, 2002; de Villiers et al., 2004; Munoz, 2006). Genital HPV is the most common sexually transmitted diseases (STDs) of viral etiology worldwide and can be categorized into 2 main groups; high risk group and low risk group, according to their oncogenic potential or type of lesion infected (zur Hausen, 2002 Munoz et al., 2003; Smith et al., 2007; Ramet et al., 2010; Gangulgy, 2012).
Approximately 16 genital HPV types; HPV 16, 18, 31, $33,35,39,45,51,52,56,58,59,66,68,73$ and 82 , have been identified as high risk type which usually associated with high grade lesions and cancer cells. HPV 16 is the most common type related to malignant tumor and their precursor lesions, whereas the low risk HPV types, such as HPV 6, 11, are mostly caused benign or non-malignant tumors and rarely found in malignancies (Walboomer et al, 1999; Munoz et al., 2003; Moody et al, 2010).

Persistent infection of high risk HPV is the majority causes for progression to high grade lesions and cervical cancer developing. Numerous epidemiological studies found that over $90 \%$ of invasive cervical cancer cells contained HPV DNA sequences (Parkin, 2002; Gao et al., 2003; Chaturvedi, 2010; Baldez da Silva et al., 2012; ICO HPV Information center, 2014). In additional, HPV 
is also reported to be associated with the other lowergenital cancers such as anus, penis, vulva, vaginal etc which showed the various incidence and prevalence of HPV infection between different region worldwide (Gao et al., 2003; Parkin, 2006; Hampl, 2007; Ramet et al., 2010; ICO HPV Information center, 2014). Although the frequency of HPV infection in other genital intraepithelial neoplasia and cancer cells are less variety than cervical cancer, but it is the interesting epidermiological data of viral caused cancer, especially vulva and vaginal including their precursor lesions which were rare incidence among all gynecological cancer. Whereas, the incidence and mortality of vulva and vaginal invasive cancers have remained stable and the evidence of HPV infection was quiet strongly increased over the past few years (ICO HPV Information center, 2014).

Vulva and vaginal cancers are rare malignancy among women worldwide, with an accounted for 27,000 and 13,000 new cases, respectively in 2008 and represented around $2 \%$ to $4 \%$ of all gynecological cancers (de Martel et al., 2012; ICO HPV Information center, 2014). Many studies reported that many risk factors of cervical cancer development, such as history of sexual behavior and other sexually transmitted diseases, smoking, alcohol consumption, history of HPV infection etc, were causally related with vulva and vaginal abnormality lesions and cancer cells (Trimble et al., 1996; Madeleine et al., 1997; Gillison, 2003). Approximately $60 \%$ of all vulva cancer cases were found in developed countries, conversely the majority of vaginal cancer could be observed in developing countries as similar to cervical cancer evidence. Vulva and vaginal cancers are common occur in elder women (>60 years) and the majority type of these cancers are squamous cell carcinoma (ICO HPV Information center, 2014; Sankaranarayanan et al., 2006; Kurunaratne, 2011).
For Thailand evidence; The Thailand Cancer Registry on 2008 reported that the incidence of vulva and Vaginal among Thai women were limited, with age standardized incidence rate (ASR) of 0.4 and 0.2 per 100,000 Thai women, respectively (Khuhaprema et al., 2013).

Numerous epidemiological studies have shown that there are two independent pathways have been associated with the progression of vulva abnormality lesions and cancer; the first pathway is classified as a differentiated keratinizing which is common occurs in elderly women. It also often differentiated vulva intraepithelial neoplasia (dVIN) and only 2\%-23\% of this type harbors HPV infection (Parkin, 2006; Trimble et al., 1996), whereas the second pathway is often affected in younger women. It is identified as non-keratinizing type (usual VIN; uVIN) which is related with warty, basaloid or mixed type of warty and basaloid. Almost of vulva intraepithelial neoplasia with non-keratinizing types tends to be associated with high risk HPV infection (Parkin, 2006; Madeleine et al., 1997; Ramet et al., 2010; Terlou et al., 2010). While, most of vaginal cancer cases, especially squamous cell carcinoma are generally attributed to HPV infection similar to cervical cancer and often occur in women over 50 years (ICO HPV Information center, 2014). Moreover, some of epidermiological studies found that women with vaginal abnormality lesions (VAIN) are more likely to have a history of cervical cancer and HPV 16 is the most common type in high-grade vaginal neoplasia and malignancy as similar to vulva and cervical cancer (Parkin, 2006; Hamp et al., 2006; de Martel et al., 2012; ICO HPV Information center, 2014).

The aim of this study was to evaluate HPV frequency and specific type-distribution from Thai women tissues with vulva and vaginal abnormality lesions and cancers.

Table 1. Human Papillomavirus Frequency of Vulva and Vaginal Cancer/Abnormal Lesion Tissues from Thai women, by Histology (n=90)

\begin{tabular}{|c|c|c|c|c|c|c|}
\hline \multirow{3}{*}{ Type of cells } & \multicolumn{3}{|c|}{ vulva } & \multicolumn{3}{|c|}{ Vaginal } \\
\hline & All cases & HPV negative & HPV positive & All cases & HPV negative & HPV positive \\
\hline & $\mathrm{n}(\%)$ & $\mathrm{n}(\%)$ & $\mathrm{n}(\%)$ & $\mathrm{n}(\%)$ & $\mathrm{n}(\%)$ & $\mathrm{n}(\%)$ \\
\hline \multicolumn{7}{|l|}{ Cancer cells } \\
\hline Squamous cell carcinoma & 30 & 16 & 14 & 4 & 2 & 2 \\
\hline Malignant melanoma & 2 & 1 & 1 & 0 & 0 & 0 \\
\hline Verrucous carcinoma & 1 & 0 & 1 & 0 & 0 & 0 \\
\hline Adenocarcinoma & 1 & 1 & 0 & 1 & 0 & 1 \\
\hline Total & $34(100)$ & $18(52.94)$ & $16(47.06)$ & $5(100)$ & $2(40)$ & $3(60)$ \\
\hline \multicolumn{7}{|l|}{ Abnormal cells (non-malignant) } \\
\hline Intraepithelial neoplasia & 1 & 0 & 1 & 3 & 0 & 3 \\
\hline Paget's disease & 2 & 2 & 0 & 0 & 0 & 0 \\
\hline $\begin{array}{l}\text { Fibroepithelial polyp/epithelial cyst } \\
\text { /Bartholin's duct cyst }\end{array}$ & 4 & 3 & 1 & 6 & 3 & 3 \\
\hline Chronic inflammation with & 8 & 4 & 4 & 19 & 10 & 9 \\
\hline \multicolumn{7}{|c|}{ hyperplasia/leukoplakin/ulcer/granulation tissues } \\
\hline the others (condyloma infection/ & 5 & 3 & 2 & 3 & 2 & 1 \\
\hline \multicolumn{7}{|l|}{ Lichen sclerosus/Leiomyoma, etc) } \\
\hline Total & $20(100)$ & $12(60)$ & $8(40)$ & $31(100)$ & $15(48.39)$ & $16(51.61)$ \\
\hline
\end{tabular}


Table 2. Human Papillomavirus Genotype Detection among Thai Women with Vulva and Vaginal Cancer or Abnormal Cytology $(n=43)$

\begin{tabular}{|c|c|c|c|c|c|c|}
\hline \multirow{3}{*}{ Type of cells } & \multicolumn{3}{|c|}{ vulva } & \multicolumn{3}{|c|}{ Vaginal } \\
\hline & All cases & single type & multiple types & All cases & single type & multiple tyoes \\
\hline & $\mathrm{n}(\%)$ & $\mathrm{n}(\%)$ & $\mathrm{n}(\%)$ & $\mathrm{n}(\%)$ & $\mathrm{n}(\%)$ & $\mathrm{n}(\%)$ \\
\hline \multicolumn{7}{|l|}{ Cancer cells } \\
\hline Squamous cell carcinoma & $14(87.5)$ & $9(56.25)$ & $5(31.25)$ & $2(66.67)$ & $1(66.67)$ & $1(33.33)$ \\
\hline Malignant melanoma & $1(6.25)$ & $1(6.25)$ & 0 & 0 & 0 & 0 \\
\hline Verrucous carcinoma & $1(6.25)$ & 0 & $1(6.25)$ & 0 & 0 & 0 \\
\hline Adenocarcinoma & 0 & 0 & 0 & $1(33.33)$ & $1(33.33)$ & 0 \\
\hline Total & $16(100)$ & $10(62.5)$ & $6(37.5)$ & $3(100)$ & $2(66.67)$ & $1(33.33)$ \\
\hline \multicolumn{7}{|l|}{ Abnormal cells (non-malignant) } \\
\hline Intraepithelial neoplasia & $1(12.5)$ & $1(12.5)$ & 0 & $3(18.75)$ & $1(6.25)$ & $2(12.5)$ \\
\hline Paget's disease & 0 & 0 & 0 & & & \\
\hline $\begin{array}{l}\text { Fibroepithelial polyp/epithelial cyst/ } \\
\text { Bartholin's duct cyst }\end{array}$ & $1(12.5)$ & 0 & $1(12.5)$ & $3(18.75)$ & $1(6.25)$ & $2(12.5)$ \\
\hline $\begin{array}{l}\text { Chronic inflammation with } \\
\text { hyperplasia/leukoplakin/ulcer/ } \\
\text { granulation tissues }\end{array}$ & $4(50)$ & $1(12.5)$ & $3(37.5)$ & $9(56.25)$ & $6(37.5)$ & $3(18.75)$ \\
\hline $\begin{array}{l}\text { the others (condyloma infection/ } \\
\text { Lichen sclerosus/Leiomyoma, etc) }\end{array}$ & $2(25)$ & $1(12.5)$ & $1(12.5)$ & $1(6.25)$ & $1(6.25)$ & 0 \\
\hline Total & $8(100)$ & $3(37.5)$ & $5(62.5)$ & $16(100)$ & $9(56.25)$ & $7(43.75)$ \\
\hline
\end{tabular}

\section{Materials and Methods}

\section{Sample collection}

Ninety formalin-fixed and paraffin-embedded (FFPE) tissues of vulva and vaginal abnormality lesion and cancers, which were collected, diagnosed during 20032012 with histological confirmed, were obtained from Specimen Bank and Pathology Department, National Cancer Institute, Thailand.

The research protocol has been reviewed and approval by Research and Ethics committee of National Cancer Institute, Thailand based on the Declaration of Helsinki and Good Clinical Practice.

\section{Sample preparation and DNA extraction}

Three to five of $5 \mu \mathrm{m}$ thick sections of vulva and vaginal tissues from FFPE samples were collected in sterile vial. Single use blade, groves and sterile forceps were used to prevent carry-over contaminant with the different samples. After deparaffinization with xylene, twice rehydration in absolute ethanol and discharge the remaining ethanol, all of samples were dried at room temperature to ensure that no residual ethanol (Ngamkham et al., 2013). Genomic DNA extraction was performed using QIAamp DNA Mini kit (QIAGEN, Germany) according to the manufacturer's instruction and genomic DNA quality was evaluated by polymerase-chain reaction with $\beta$-globin primers (Jacob et al., 1996); sense 5'-ACA CAA CTG TGT TCA CTA GC-3' and antisense 5'-GAA ACC CAA GAG TCT TCT CT-3'. Total volume of $25 \mu \mathrm{l}$ PCR mixture was consisted of $5 \mu \mathrm{l}$ extracted DNA, $0.4 \mu \mathrm{M}$ of sense and antisense primers, $2.5 \mathrm{U}$ Taq polymerase, $200 \mathrm{nM}$ of each dNTPs and $1.5 \mathrm{mM} \mathrm{MgCl} 2$. The amplification was performed using GeneAmp 9700 thermal cycler (Applied Biosystemic, USA) and cyclic condition under the following; initial denaturation step of $94^{\circ} \mathrm{C}, 5 \mathrm{~min}$, follow by 40 cycles of $94^{\circ} \mathrm{C}, 1 \mathrm{~min} ; 60^{\circ} \mathrm{C}, 1 \mathrm{~min} ; 72^{\circ} \mathrm{C}, 1 \mathrm{~min}$ and re-extension at $72^{\circ} \mathrm{C}$ for $5 \mathrm{~min}$ in the final step. Amplified products were visualized on $2 \%$ agarose gel electrophoresis with UV transilluminator (SYNGENE, England).

\section{HPV DNA detection and genotyping}

HPV DNA detection and genotyping method were performed according to Jacob et al. (1997; 2000). HPV DNA was amplified by polymerase chain reaction (PCR) using general primers GP5+/6+(GP5+; 5'-TTT GTT ACT GTG GTA GTA ACT AC-3' and GP6+ biotin-labeled 5'-GAA AAA TAA ACT GTA AAT CAT ATT C-3') to amplify the corresponding part of HPV L1 gene and DNA from the HPV 16 positive SiHa cell line was used as a positive control. The PCR mixture contained $10 \mu \mathrm{l}$ extracted DNA, $0.4 \mu \mathrm{M}$ of each specific primer, $2.5 \mathrm{U}$ Taq polymerase, $200 \mathrm{nM}$ of each dNTPs and $1.5 \mathrm{mM} \mathrm{MgCl}_{2}$ and PCR condition were amplified following profile of initial denature at $94^{\circ} \mathrm{C}, 5 \mathrm{~min}$ and then followed by 40 cycles of $94^{\circ} \mathrm{C}, 1 \mathrm{~min} ; 38^{\circ} \mathrm{C}, 2 \mathrm{~min} ; 72^{\circ} \mathrm{C}, 1.30 \mathrm{~min}$. The finally step was re-extension for $4 \mathrm{~min}$ at $72^{\circ} \mathrm{C}$. HPV genotype was identified by using Enzyme-immunoassay (EIA) with HPV specific probes for high risk (HPV 16, $18,31,33,35,39,45,51,52,56,58,59,66$, and 68$)$ and low risk HPV types (HPV 6, 11, 26, 34, 40, 42, 43, 44, $53,54,55,57,61,70,71,72,73,81,82,83,84$, and 89), In brief, mixer solution of $5 \mu \mathrm{HPV}$-positive PCR product and $50 \mu \mathrm{l} 1 \mathrm{xSCC}, 0.5 \%$ Tween 20 was added to each well of streptavidin-coated plate (Roche, Germany) and incubated at $37^{\circ} \mathrm{C}$ for $60 \mathrm{~min}$ to completely bind biotinylated compounds. Unbound biotinylated were eliminated by three times washing with freshly prepared $1 \mathrm{xSCC}$, $0.5 \%$ Tween 20 solution. Then, digoxigenin labeled HPV specific probes was added and incubated for $60 \mathrm{~min}$ at 
$37^{\circ} \mathrm{C}$ to allow hybridization and unbound compounds were discharged by washing. HPV specific type detection was achieved by adding freashly prepared conjugate solution (Roche, Germany) and substrate; p-Nitrophenyl phosphate (Sigma, USA), respectively. The optical density (405nm and $620 \mathrm{~nm}$ ) was twice measured at 16-24 hours.

\section{Results}

A total of ninety formalin-fixed and paraffin-embedded (FFPE) samples from Thai women with vulva or vaginal cancer/abnormal lesions were collected. Theses samples consisted of 54 samples of vulva tissues and 36 samples of Vaginal tissues. All of samples had histological confirmed by Pathology Division, National Cancer Institute, Thailand. The mean ages of vulva and Vaginal patients were $59.36 \pm 4.64$ years and $53.44 \pm 11.25$ years, respectively. Squamous cell carcinoma was found the most frequency in both types of cancers. Of 34 vulva cancer samples can be categorized to 30,2,1 and 1 samples of squamous cell carcinoma, malignant melanoma, verrucous carcinoma and adenocarcinoma, respectively. While 20 samples of vulva abnormal cytology were classified into intraepithelial neoplasia (1/20), Paget's disease (2/20), fibroepithelial polyp (4/20) etc. whereas, 4/5 (80\%) and $1 / 5(20 \%)$ samples of Vaginal cancer were squamous cell carcinoma and adenocarcinoma, respectively, as represented in Table I

HPV DNA detection in cancer and abnormal lesion samples by PCR-EIA methods were observed that $47.06 \%$ (16/34) of vulva cancer and 40\% (8/20) of vulva abnormal lesion samples shown HPV positive. About 60\% (3/5) and $51.61 \%(16 / 31)$ of Vaginal cancers and abnormal lesion samples, respective could detect HPV DNA, as shown in Table I.

From our HPV genotype results found that single type and multiple type infection could be detected in both type of cancer or abnormal lesion samples in the different histological categorizes. In additional, the frequency of

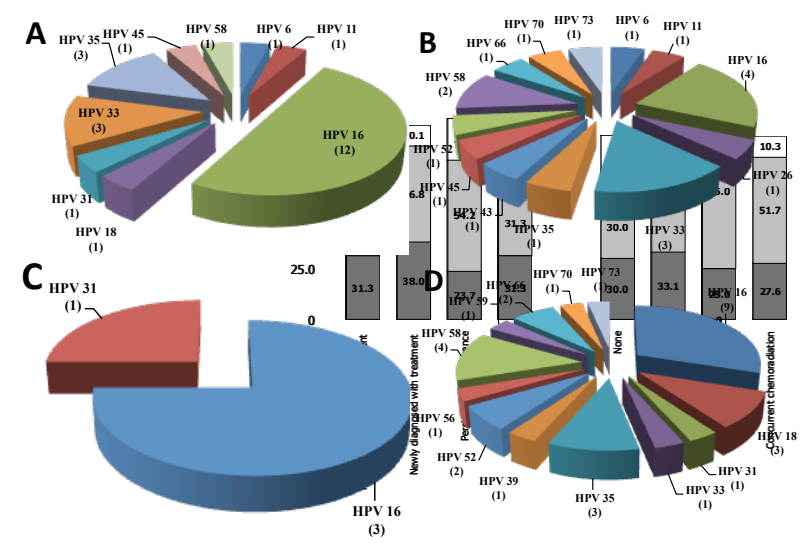

Figure 1. Overall Human Papillomavirus Type Distribution in Vulva Cancer Cells $(n=16)(A)$, Vulva Cells with Abnormal Cytology (n=8) (B), Vaginal Cancer Cells $(n=3)(C)$ and Vaginal Cells with Abnormal Cytology (n=16) (D) from Thai Women $(n=43)$ (included single and multiple HPV types infection) single was higher than multiple type infection and also detected in cancer cells higher than abnormal cytology lesion samples, as shown in Table II.

The most common HPV type in this study was HPV 16 , accounting for $75 \%$ (12/16 samples, in which 7/16 cases were single type infection and $5 / 16$ cases were multiple type infection) and $100 \%$ (3/3 samples; consisted of $2 / 3$ and $1 / 3$ were single and multiple type infection, respectively) of vulva and Vaginalcancers, respectively. Moreover, the results of genotype in abnormal lesion, HPV 16 were higher frequency than the other types. It accounted 4/8 and 9/16 samples of all vulva and vaginal abnormal lesions with HPV positive, respectively. The three common frequency type which could be detected in this study were HPV 16,33 and 35 for vulva cancer cells and HPV 16,33 and 58 for vulva abnormal cells, whereas HPV 16 and 31 were found in Vaginal cancer cells and HPV 16, 58, 18 and 35 were the top three frequency in Vaginal abnormal cytology lesion cells. Almost of HPV type detection samples belong to the high risk type, but the low risk type, such as HPV 6, 11 which were usually found in lower genital abnormal lesion or benign tumor, were also detected in vulva samples as co-infection with the other high risk type; such as HPV6/11/16/33, HPV $6 / 16$ etc. However, HPV genotype in abnormal cytology lesion samples showed a wide variety of type distribution and could be detected both of high risk and low risk type, as shown in Figure I

\section{Discussion}

Vulva and Vaginal cancers are rare gynecological cancer among women worldwide and common diagnosed in older women (over 65 years). There are many epidemiological risk factors, included persistent high risk HPV infection, were essential cause for vulva and Vaginal abnormal lesions developing or cancer cells progression (ICO HPV Information center, 2014; Karunaratne, 2011; Bharti wt al., 2010). Although high risk HPV infection is a well-established cause of cervical cancer, but there is now growing evidence of HPV being a relevant factor in vulva and Vaginal cancers. HPV infection is associated with both of benign and malignant lesions and almost detected in the younger women rather than elder women (zur Hausen, 2002; De Vuyst et al, 2009; Ramet et al., 2010; Dittmer et al., 2011)

In this study, HPV DNA detection was performed on section of paraffin-embedded biopsy. The frequency of HPV positive was detected to be $47.06 \%$ and $60 \%$ in vulva and Vaginal cancers, respectively. It was not different from results of a systematic review which was conducted by Smith et al. (2007) in which $40.1 \%$ and $65.5 \%$ of vulva and vaginal cancer samples, respectively, presented HPV DNA. While the meta-analysis of HPV prevalence in vulva, Vaginal and anal from 93 studies which was investigated by De Vuyst et al. (2009) reported the overall prevalence of vulva and Vaginal carcinoma were $40.4 \%$ and $69.9 \%$, respectively. However, the HPV frequency among women with abnormal cells or precursor lesions of vulva (40\%) and Vaginal $(51.61 \%)$ in this study were lower than the reported of Smith et al. (2007) and De 
Vuyst et al (2009) which represented HPV prevalence approximately $60 \%-85 \%$ and $90 \%$ among vulva and Vaginal intraepithelial neoplasia, respectively, suggested that vulva and Vaginal sample with abnormal lesions in this study did not specific recruited only abnormal cytology tissues with intraepithelial lesions (VIN or VAIN) as similar to the criteria of two those previous studies. All of these tissues with abnormal cytology were random investigated in various histopathology types such as chronic inflammation, Leiomyoma etc, including intraepithelial neoplasia and some of vulva tissues with keratinizing histological type such as lichen sclerosus was likely to be non-HPV-related and usually found in older women. (ICO WHO Information center, 2014; Smith et al., 2007; Madeleine et al., 1997) Furthermore, not all of vulva and Vaginal cancers are induced by HPV, it dependent on the several factors such as age, cancer history, type of cancer diagnosed, etc (Hampl et al., 2006). However, our results of HPV DNA showed that $1 / 1$ sample of vulva intraepithelial neoplasia (VIN) and 3/3 samples of Vaginal intraepithelial neoplasia (VAIN) in this study found HPV infection.

HPV 16 is the most common genotype identified in this study as similar to the other reports (ICO WHO Information center, 2014; Ramet et al., 2010; Giuliano et al., 2008; Hampl et al., 2007). It represented 12/16 (75\%), $4 / 8(50 \%), 3 / 3(100 \%)$ and 9/16 (56.25\%) in vulva cancer, vulva abnormal lesions, Vaginal cancer and Vaginal abnormal lesion samples, respectively. The most three common HPV types which was detected in vulva cancer and abnormal lesions were 16,33, 35 and 16, 33, 58, respectively whereas HPV 16, 33 and HPV 16, 58, 18,35 were found higher frequency than the other types in Vaginal tissues with cancer and abnormal lesions, respectively. And we observed that HPV 18 could be detected as co-infection with other high risk types and all of low risk type was never found as single infection in this study. Although the most three common HPV typedistribution in vulva and Vaginal in our study showed slightly different from the other reports (Ramet et al, 2010; ICO WHO Information center, 2014; Hampl et al., 2007; Baldez da Silva et al., 2012), due to the different HPV detection methods, amount or type of sample collection, population characteristic, etc, but there are several studied reported which found that high risk HPV type such as HPV $16,18,31,33,35,45,51,52,56$ or 58 have been associated with vulva, vagina, penis, anus or cervical cancers and abnormal lesions (Giuliano et al., 2008; Baldez da Silva et al., 2012; Hampl et al., 2007; De Vuyst et al., 2009). Furthermore, some of the less common HPV types, such as HPV 43 and HPV 73 were observed in vulva and Vaginal with abnormal lesions, but none of the cancer cells harbored these types. HPV types in vulva and Vaginal tissues with abnormal cytology were more variety than cancer cells and have been detected both of high risk and low risk type infection. While most of high risk types were found in cancer cells, suggested that low risk HPV type infection in vulva or vaginal cancers might be transient infection and did not the major cause of cancer cell development.

However, there are some limitation of this study was that the small sample size of vulva and Vaginal cases, especially Vaginal cancer samples, because of some of Vaginal samples, which had recurrent from cervical cancers or other cancers were excluded. Moreover, approximately $10 \%-15 \%$ of vulva and Vaginal cases were referred from the other cancer centers or hospitals to diagnose confirmed that lead to lose some patients' data and small amount of tissues were not enough to HPV test. For this point, HPV DNA yield could be improved by increasing the number or larger size of sample tissues. Otherwise, the variety of population group, sample selection and histological diagnosis results were to be the important factors which were used to be considerable (Hampl et al., 2006; Giuliano et al., 2008)

This finding demonstrated that HPV 16 was the most common HPV type present in vulva and Vaginal tissues with abnormal cytology lesions and cancer cells while HPV 18 and the other types showed less frequently for both organs from Thai women and the HPV type distribution of vulva and Vaginal were not variety as cervical cells. However, it is interesting baseline data on HPV prevalence of vulva and Vaginal abnormal/cancer from Thai women, which there are limited evidences available and both of these cancers are more less frequency compared to cervical cancer, although the sample size in this study was small and did not represented overall incidence and prevalence of Thai women population. Moreover, persistent high risk HPV infection is the effective factor that causes the abnormal or cancer cells of genital tract development, the efficiency prevention programs which reinforce the HPV detection and vaccination may decrease the incidence of $\mathrm{HPV}$-associated diseases in the nearly future.

\section{References}

Baldez da Silva MF, Guimaraes V, Silva MAR, et al (2012). Frequency of human papillomavirus type 16,18,31 and 33 and site of cervical lesions in gynecological patients from Recife, Brazil. Genet Mol Res, 11, 462-6.

Bharti AC, Shukla S, Mahata S et al, (2010). Human papillomavirus and control of cervical cancer in India. Expert Rev Obstet Gynnecol, 5, 329-46.

Chaturvedi AK (2010). Beyond cervical cancer: burden of other HPV-related cancer among men and women. $J$ Adolesc Health, 46, 20-6.

de Martel C, Ferlay J, Franceschi S, et al (2012). Global burden of cancers attributable to infections in 2008: a review and synthetic analysis, Lancet Oncol, 13, 607-15.

de Villiers EM(2001). Taxonomic classification of papillomavirus. Papillomavirus Rep, 12, 57-67.

de Villiers EM, Fauquet C, Broker TR, et al (2004). Classification of papillomaviruses. Virol, 324, 17-27.

De Vuyst H, Clifford GM, Nascimento MC, et al (2009). Prevalence and type distribution of human papillomavirus in carcinoma and intraepithelial neoplasia of the vulva, Vaginal and anal: a meta-analysis. Int J Cancer, 124, 1626-36.

Dittmer C, Katalinic A, Mundhenke C, et al (2011). Epidemiology of vulvar and vaginal cancer in Germany. Arch Gynecol Obstet, 284, 169-74.

Ganguly N (2012). Human papillomavirus-16 E5 protein: oncogenic role and therapeutic value. Cell Oncol, 35, 67-76.

Gao W, Weng J, Ga Y et al (2013). Comparison of the vaginal microbiota diversity of women with and without human 
papillomavirus infection: a cross-section study, BMC Infectious Diseases, 13, 271 (open access).

Gillison ML, Shah KV (2003). Chapter 9: Role of Mucosal Human Papillomavirus in Nongenital Cancers, J Natl Cancer Inst Monogr, 31, 57-65.

Hampl M, Sarajuuri H, Wentzensen N, et al (2006). Effect of human papillomavirus vaccines on vulvar, vaginal and anal intraepithelial lesions and vulvar cancer. Obstet Gynecol, 108, 1361-8.

Hampl M, Wentzensen N, Vinokurova S, et al (2007). Comprehensive analysis of 130 multicentric intraepithelial female lower genital tract lesions by HPV typing and p16 expression profile. J Cancer Res Clin Oncol, 133, 235-45.

ICO Information Center on HPV and Cancer (HPV Information Center) (2014). Human Papillomavirus and Related Diseases Report.

Jacob MV, van der Brule AJ, Snijder PJ, et al (1996). A nonradioactive PCR enzyme-Immunoassay enables a rapid identification of HPV 16 and 18 in cervical scrapes after GP5+/6+ PCR. J Med Virol, 49, 223-9.

Jacob MV, van der Brule, Snijder PJ, et al (1997). A general primer GP5+/6(+)-mediated PCR-enzyme immunoassay method for rapid detection of 14 high-risk and 6 low-risk human papillomavirus genotypes in cervical scrapings. $J$ Clin Microbiol, 35, 791-5.

Jacob MV, Walboomers JM, Snijder PJ, et al (2000). Distribution of 37 mucosotropic HPV types in women with cytologically normal cervical smears: the age-related pattern for high-risk and low-risk types. Int J Cancer, 87, 221-7.

Karunaratne K (2011). Review: squamous cell vulva cancer. Sri Lanka J Obstet Gynecol, 33, 30-2.

Khuhaprema T, Attasara P Sriplung H, et al (2013). Cancer in Thailand Vol VII 2007-2008, Bangkok, Thailand.

Madeleine MM, Daling JR, Carter JJ, et al (1997). Cofactor with human papillomavirus in a population-based study of vulva cancer. J Natl Cancer Inst, 39, 1516-23.

Moody CA, Laimins LA (2010). Human papillomavirus oncoproteins pathways to transformation. Nature Reviews, 10,550-60.

Munoz N, Bosch FX, de Sanjose S, et al (2003). Epidemiologic classification of human papillomavirus types associated with cervical cancer. $N$ Engl Med, 348, 518-27.

Munoz N, Castellsague X, de Gonzalez AB, et al (2006). Chapter I: HPV in the etiology of human cancer vaccine, 24, 1-10.

Ngamkham J, Homcha-aim P, Boonmark K, et al (2013). Preliminary study on human papillomavirus frequency and specific type-distribution in vulva cancer from thai women. Asian Pac J Cancer Prev, 14, 2355-9.

Parkin DM (2006). The global health burden of infection associated cancer in the year 2002. Int J Cancer, 118, 3030-44.

Ramet J, van Esso D, Meszner Z, et al (2010). Position paperHPV and the primary prevention of cancer, improving vaccine uptake by paediatricians. Eur J Pediatr, 170,309-21.

Sankaranarayanan R, Feray J (2006). Worldwide burden of gynecological cancer: the size of the problem. Best Pract Res Clin Obstet Gynaecol, 20, 207-25.

Smith JS, Lindsay L, Hoots B, et al (2007). Human papillomavirus type distribution in invasive cervical cancer and high-grade cervical lesion: a meta-analysis update. Int J Cancer, 121, 621-2.

Terlou A, Blok LJ, Helmerhorst TJM, et al (2010). Premalignant epithelial disorders of the vulva: squamous vulvar intraepithelial neoplasia, vulvar Paget's disease and melanoma in situ. Acta Obstet Gynecol, 89, 741-48.

Trimble CL, Hildesheim A, Brinton LA, et al (1996). Heterogeneous etiology of squamous carcinoma of the vulva. 